\title{
Psychological distress as a risk factor for all-cause, chronic disease- and suicide-specific mortality: a prospective analysis using data from the National Health Interview Survey
}

\author{
Meghan Hockey ${ }^{1}$. Tetyana Rocks ${ }^{1} \cdot$ Anu Ruusunen ${ }^{1,2,3}$. Felice N. Jacka ${ }^{1}$. Wentao Huang ${ }^{4} \cdot$ Bing Liao $^{4}$. \\ Dagfinn Aune $\mathrm{e}^{5,6,7,8} \cdot$ Yafeng Wang $^{9} \cdot$ Jing $\mathrm{Nie}^{10}$ (1) $\cdot$ Adrienne $\mathrm{O}^{\prime} \mathrm{Neil}^{1}$
}

Received: 21 November 2020 / Accepted: 2 June 2021 / Published online: 7 August 2021

(c) Springer-Verlag GmbH Germany, part of Springer Nature 2021

\begin{abstract}
Purpose The risk psychological distress (PD) confers on mortality due to specific chronic diseases compared to suicide is unclear. Using the National Health Interview Survey (NHIS), we investigated the association between PD levels and risk of all-cause and chronic disease-specific mortality and compared the contribution of chronic disease-related mortality to that of suicide.

Methods Data from 195, 531 adults, who participated in the NHIS between 1997 and 2004, were linked to the National Death Index records through to 2006. Multivariable Cox regression models were used to estimate hazard ratios (HRs) and their 95\% confidence intervals (CIs) across four levels of PD, measured using the Kessler-6 scale. Outcomes included allcause mortality, and mortality due to all CVDs and subtypes, all cancers and subtypes, diabetes mellitus, alcoholic liver disease and suicide.

Results During a mean follow-up time of 5.9 years, 7665 deaths occurred. We found a dose-response association between levels of PD and all-cause mortality, with the adjusted HRs (95\% CI) elevated for all levels of PD, when compared to asymptomatic levels: subclinical 1.10 (1.03-1.16), symptomatic 1.36 (1.26-1.46) and highly symptomatic 1.57 (1.37-1.81). A similar association was found for all CVDs and certain CVD subtypes, but not for cancers, cerebrovascular diseases diabetes mellitus. Excess mortality attributable to suicide and alcoholic liver disease was evident among those with levels of PD only. Conclusion PD symptoms, of all levels, were associated with an increased risk of all-cause and CVD-specific mortality while higher PD only was associated with suicide. These findings emphasise the need for lifestyle interventions targeted towards improving physical health disparities among those with PD.
\end{abstract}

Keywords Psychological distress $\cdot$ Mortality $\cdot$ Cardiovascular disease $\cdot$ Suicide $\cdot$ Cancer $\cdot$ National Health Interview Survey

Jing Nie and Adrienne O’Neil should be considered joint senior author.

Jing Nie

njamm.5818@stu.xjtu.edu.cn

1 IMPACT (The Institute for Mental and Physical Health and Clinical Translation), The Food and Mood Centre, Deakin University, Geelong, Australia

2 Institute of Public Health and Clinical Nutrition, University of Eastern Finland, Kuopio, Finland

3 Department of Psychiatry, Kuopio University Hospital, Kuopio, Finland

4 School of Nursing, Guangdong Pharmaceutical University, Haizhu District, Guangzhou, China

5 Department of Epidemiology and Biostatistics, School of Public Health, Imperial College London, London, UK
6 Department of Nutrition, Bjørknes University College, Oslo, Norway

7 Department of Endocrinology, Morbid Obesity and Preventive Medicine, Oslo University Hospital, Oslo, Norway

8 Unit of Cardiovascular and Nutritional Epidemiology, Institute of Environmental Medicine, Karolinska Institute, Stockholm, Sweden

9 Department of Epidemiology and Biostatistics, School of Health Sciences, Wuhan University, Wuhan, China

10 Department of Sociology, Institute for Empirical Social Science Research, School of Humanities and Social Sciences, Xi' an Jiaotong University, 28 Xianning West Rd, Xi' an 710049, Shaanxi, China 


\section{Introduction}

Mental disorders are highly prevalent and a leading contributor to global disability [1]. Whilst debilitating in their own right, mental disorders often co-occur with physical health comorbidities with up to $80 \%$ of those living with a serious mental disorder (SMD) reporting a physical health condition [2]. Comorbid conditions (e.g. cardiovascular diseases) reduce quality of life [3], impede employment and recovery [4] and increase the risk of premature mortality $[2,5]$. In particular, individuals with Severe Mental Disorders (SMDs) have an average reduced life expectancy of 20 years [6]. This may in part be explained by the excess risk of suicide-related mortality, which is well recognized among those with mental disorders [7, 8]. However, emerging evidence suggests this mortality gap is also largely explained by poor physical health [2]. Indeed, recent Australian data show that more than three quarters of this excess mortality are attributable to the impact of chronic physical health conditions, such as cardiovascular disease (CVD) and cancer, rather than suicide [9]. This can be explained by the illness itself, but also by the noxious effects of medications and poor lifestyle behaviors associated with SMDs, including higher smoking rates, physical inactivity and increased alcohol consumption [10].

The 'common mental disorders', depression and anxiety, are similarly associated with an increased prevalence of chronic health conditions (e.g. CVD) [2]. However, there is an apparently differential impact of these conditions on mortality. Previous large population studies have shown that depression is a risk factor for all major disease-related causes of death [11]. Conversely, anxiety appears to be somewhat protective $[11,12]$. Possibly due to these differential impacts, the association of comorbid depression and anxiety symptoms (i.e. psychological distress) to mortality is less clear [11,13], suggesting a need for further research.

Psychological distress (PD) is characterized by symptoms of depression and anxiety that typically co-exist [14]. These symptoms can range in severity, but are often subthreshold and do not meet criteria for a clinical diagnosis [15]. In common with other mental health conditions, PD is associated with unhealthy lifestyle behaviors, such as poor diet, smoking, physical inactivity, and alcohol intake, that may predispose individuals to chronic diseases [16]. For instance, clinically significant levels of PD are associated with an increased risk of incident CVD [17], chronic obstructive pulmonary disease and diabetes [18]. Moreover, prospective studies have observed associations between PD and an increased risk of mortality due to chronic diseases such as CVD [19, 20], cancer [21] and liver disease [22]. More recently, a prospective study, which examined data (1997-2009) from the National Health Interview Survey (NHIS) $(N=330,367)$, observed an association between
PD and all-cause, CVD and cancer-specific mortality [20]. However, the specific causes of mortality, such as subtypes of CVD (e.g. heart failure, cerebrovascular disease), were not examined. Moreover, although high levels of PD are an established risk factor for death by suicide [23], few studies have considered the risk PD confers to mortality that is specific to both chronic diseases and suicide, especially across the spectrum of PD symptom severity.

Therefore, using data from the NHIS $(N=195,531)$, this study aimed to address these gaps by estimating the risk PD confers on premature mortality and specific causes of mortality including chronic diseases (i.e. all CVDs, cancers, diabetes mellitus and alcoholic liver disease), specific disease subtypes and suicide.

\section{Methods}

\section{Study design and population}

This prospective cohort study examined data from the NHIS. The NHIS is an ongoing US cross-sectional survey that has been conducted annually by the National Centre for Health Statistics in collaboration with the US Census Bureau. The NHIS uses a stratified, multistage sample design to select participants to capture a nationally representative sample of the US civilian non-institutionalized population. Information is collected through face-to-face household interview surveys with all household members (for basic health information only) and one randomly sampled adult and child (for additional health information). More details on the study methodology can be found elsewhere [24]. The publicuse survey data from the NHIS are available from 1956 to 2019. Since 1997, the NHIS included the information of psychological distress measured by the Kessler- 6 scale. The public-use mortality data for detailed death causes (such as suicide, ischaemic heart disease, heart failure, etc.) were available up to 2006. Therefore, for the current study, data from eight waves of the NHIS conducted from 1997 to 2004, including mortality data up to 2006, were used. Data from 237,847 participants who were aged $18-84$ years were used and linked to mortality data for all-causes, specific chronic diseases and suicide $[25,26]$. We excluded 3307 participants with missing data on PD, and 39,009 participants with history of diabetes, liver disease, coronary heart disease, stroke or cancer at baseline, to exclude the possibility that the presence of disease at baseline was driving any excess mortality outcomes observed at follow-up. This left 195, 531 participants available for analyses. The NHIS has been reviewed and approved by the Institutional Review Board at the Centre for Disease Control and Prevention. Written informed consent was obtained from all participants. 


\section{Exposure (psychological distress)}

Psychological distress was measured using the Kessler-6 scale (K6), a standardized screening instrument developed specifically for use in general population surveys [27]. The K6 comprised six questions that asked respondents how often they felt sad, nervous, restless or fidgety, hopeless, that everything was worth an effort, and/or worthless during the past 30 days. Responses were scored on a 5-point Likert scale that ranged in frequency from "all of the time" (score of 4) to "none of the time" (score of 0 ), with total scores ranging between 0 and 24 . A cut-off point of $\geq 6$ has previously been identified for "moderate" psychological distress based on treatment seeking patterns in a population sample [28]. Based on these cut-off points, four categories of psychological distress were identified for this study: asymptomatic, score $=0$; subclinical, scores $=1-5$; symptomatic, scores $=6-12$; and highly symptomatic, scores $=13-24$ $[29,30]$. For the secondary analysis, a cut-off score of 13 was used to identify individuals with and without serious psychological distress (SPD) [27] (i.e. scores equivalent to those within the highly symptomatic distress category). This method is in line with previous studies and was used to enable comparability with the wider literature [31]. For example, on the basis of ROC curve analysis, a score of $\geq 13$ on K6 has previously been selected as the optimal cutpoint to identify psychological distress associated with serious mental illness [32]. This cutpoint is associated with a sensitivity of 0.36 , a specificity of 0.92 , and a total classification accuracy of 0.92 and equalizes the number of false positives and false negatives in population samples [27].

\section{Outcome (mortality)}

Data from NHIS participants, collected between 1997 and 2004, were linked to the National Death Index (NDI) records up to 2006. The NDI commenced in 1979 by the National Centre for Health Statistics as a centralized database of all US deaths and has been validated for all-cause and causespecific mortality information [33]. Causes of death were classified using the 10th revision of the International Statistical Classification of Diseases, Injuries, and Causes of Death (ICD-10), and grouped into the following categories: cancers, diabetes mellitus, CVDs, digestive diseases and self-harm and interpersonal violence. For the purpose of this study, the primary outcome was all-cause mortality, and secondary outcomes were mortality arising due to specific causes of mortality, including chronic diseases (i.e. CVD, diabetes, cancer and alcoholic liver disease), chronic disease subtypes and suicide. Deaths from all CVDs were coded using codes I11, I13, I10, I12, I70, I71-I78 and I80-I99, including ischaemic heart disease (I20-I25), heart failure (I50), and cerebrovascular disease (I60-I69). All cancer-specific deaths were identified using codes $\mathrm{C} 00-\mathrm{C} 97$, which included cancer of the trachea, bronchus, and lung mortality (C33-C34), cancer of the colon, rectum and anus (C18-C21), cancer of the breast (C50) and cancer of the prostate (C61). Mortality due to diabetes mellitus was identified by code E10-E14 and alcoholic liver disease by code K70. Finally, suicide-specific mortality was identified using codes X72-X74, X60-X71, X75-X84 and Y87.0.

\section{Covariates}

Potential confounders were selected a priori based on evidence demonstrating potential to confound the relationship between PD and mortality. Demographic variables such as age, sex, race (Hispanic, non-Hispanic White, non-Hispanic Black, and Other), education level (less than high school degree, high school degree, more than high school degree) and income level (family income to poverty ratio at or below the federal poverty level $(\leq 1)$, middle income $(>1$ and $<4)$, or high income $(\geq 4)$ ) were considered for inclusion. Lifestyle-related variables such as body mass index (BMI) $\left(<25 \mathrm{~kg} / \mathrm{m}^{2}, 25-30 \mathrm{~kg} / \mathrm{m}^{2},>30 \mathrm{~kg} / \mathrm{m}^{2}\right)$, physical activity (meeting or not meeting recommended physical activity guidelines of 150 min per week), smoking status (never, former, current), and alcohol drinking (lifetime abstainer, former drinker, current drinker) were also considered for inclusion.

\section{Statistical analyses}

Baseline characteristics of the participants were presented as percentages (\%). Chi-square tests were used to assess the differences in baseline characteristics across the four PD levels $(0,1-5,6-12,13-24)$. Cox proportional hazards models were used to calculate the hazard ratios (HRs) and 95\% confidence intervals (CIs) for associations between PD and mortality, with participants scoring " 0 " (i.e. asymptomatic) as the referent group. HRs were unadjusted and maximally adjusted (age, sex, race, education, income, BMI, alcohol drinking, physical activity, smoking status and survey year). To take into account potential reverse causation, sensitivity analyses were also conducted by excluding the first two years of follow-up. In the secondary analyses, we evaluated the association of PD with mortality, comparing SPD (scores 13-24) versus no SPD (scores 0-12) in the maximally adjusted model. Subgroup analysis based on age, sex, smoking status and physical activity were conducted among those with and without SPD for the primary outcome (all-cause mortality); a small number of cases prohibited subgroup analyses for all other outcomes. The NHIS uses a multistage sampling design; strata, cluster and weights were accounted for in the analyses for generalization of the estimates. All analyses were conducted with Stata version 
Table 1 Baseline characteristics of individuals according to levels of psychological distress $(N=195,531)$

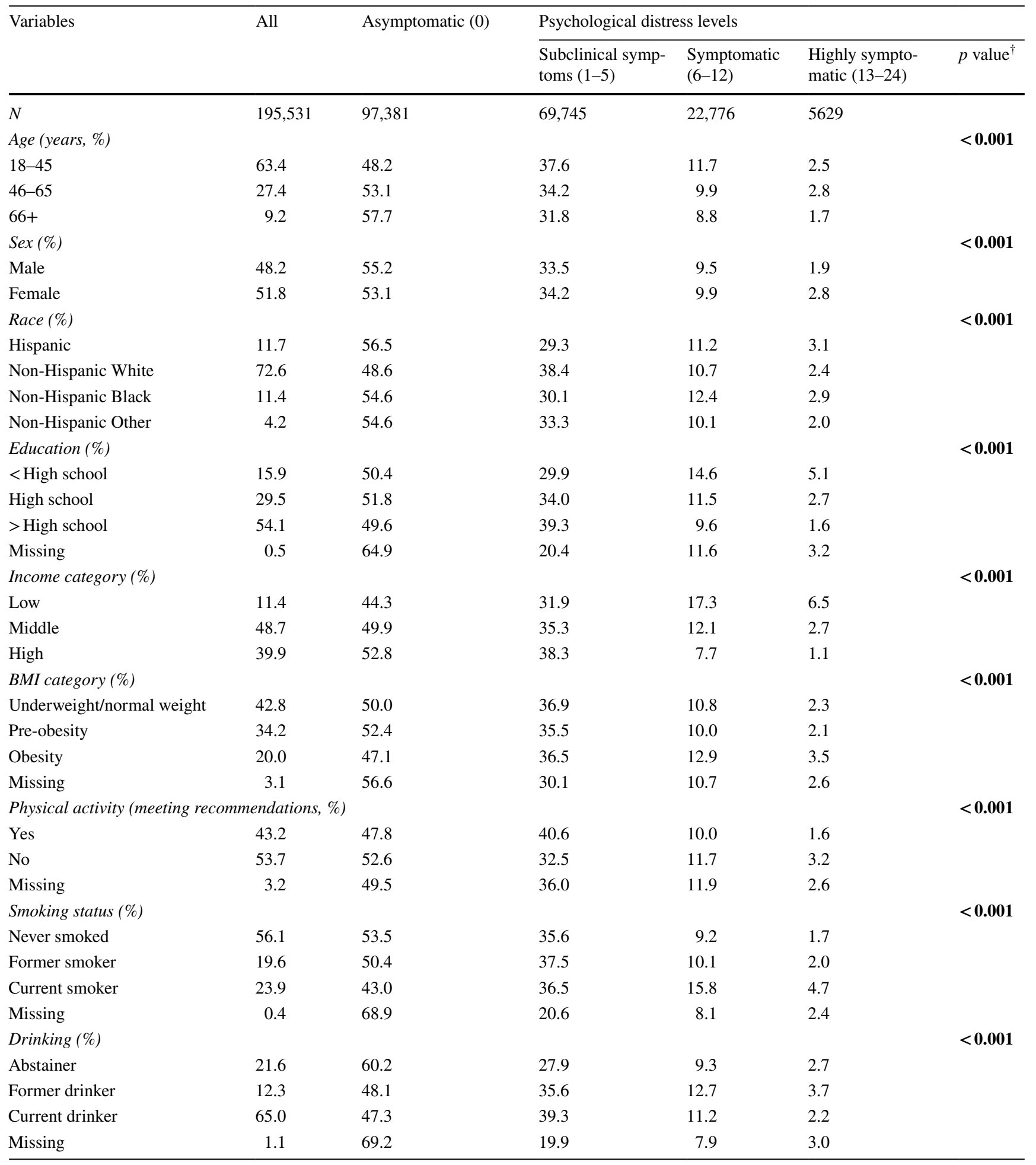

Values rounded to 1 decimal place BMI, body mass index

${ }^{\dagger}$ Calculated using Chi-square tests 
13.0 (Stata Corp, College Station, TX, USA). A two-tailed $p$ value of $<0.05$ was considered statistically significant.

\section{Results}

A total of 195, 531 participants were included in this analysis $(51.8 \%$ women). More than half experienced PD symptoms: $49.8 \%$ were asymptomatic, $35.7 \%$ were subclinically symptomatic, $11.6 \%$ were symptomatic and $2.9 \%$ were highly symptomatic. In general, females, those with low education or income, those who did not meet physical activity recommendations, current smokers and former drinkers reported higher levels of distress (see Table 1).

\section{All-cause mortality}

During a mean follow-up time of 5.9 years, a total of 7665 deaths occurred. The leading causes of death across the cohort were chronic diseases, namely CVD $(N=2321)$ and cancers $(N=1872)$. When considering all participants, $56.4 \%$ of deaths were due to chronic diseases (i.e. CVD, cancer, diabetes and alcoholic liver disease) compared with $1.7 \%$ by suicide and $41.9 \%$ by other causes (e.g. infections or nervous system disorders). In those with any level of PD symptoms, $56.4 \%$ of deaths were due to chronic diseases and $1.7 \%$ due to suicide. In the unadjusted model, subclinical symptoms conferred a decreased risk for allcause mortality, whereas this risk was elevated for those who had symptomatic and highly symptomatic levels of PD (see Table 2). However, after adjustment for covariates, the association between PD and all-cause mortality occurred in a dose-response fashion whereby mortality risk increased along with PD symptom severity. This was equivalent to a $10 \%, 36 \%$ and $57 \%$ increased risk of all-cause mortality among those with subclinical, symptomatic and highly symptomatic levels of PD, respectively (see Fig. 1).

\section{Cause-specific mortality}

Similar dose-response trends were observed for exposure to PD and CVD-specific mortality. In the adjusted model, those with highly symptomatic levels of PD had an $86 \%$ increased risk of CVD-specific mortality (see Fig. 1). Further investigation of specific causes showed that those with elevated levels of PD were at increased risk of mortality due to ischaemic heart disease and heart failure, whereas there was no association between PD and cerebrovascular disease. In unadjusted models, subclinical symptoms, but not symptomatic or highly symptomatic levels of $\mathrm{PD}$, were associated with decreased risk of all-cause cancer mortality. However, after adjustment for covariates, this association was attenuated. Similarly, in adjusted models, no association was observed between any level of PD and cancer of the colon, rectum and anus, cancer of the trachea, bronchus and lung, cancer of the prostate and cancer of the breast. Moreover, in the crude and adjusted analysis, no association was observed between PD and diabetes mellitus mortality. Among those in the highest distress category, the HR for alcoholic liver disease exceeded that of all chronic diseases. However, the association between PD and mortality due to alcoholic liver disease was only evident among those with highly symptomatic levels of PD. A dose-response association was also observed between the spectrum of PD symptoms and suicide-specific mortality. However, this association was only statistically significant among those with highly symptomatic levels of PD (see Fig. 1).

\section{Sensitivity analysis}

To minimize the potential for reverse causation, we conducted a sensitivity analysis excluding participants during the first 2 years of the follow-up $(N=1683$, see Table 3$)$. The results showed a pattern that was mostly similar to that found in the main analysis. However, after the exclusion of these participants, no association was observed between PD and suicide mortality across all levels of PD.

\section{Secondary analysis}

We re-ran our analyses dichotomising K-6 scores to compare those with and without SPD. The results were largely comparable to those of the main analysis (see Supplementary Table 1). In adjusted models, those with SPD had an increased risk of all-cause mortality when compared to those without SPD. When comparing specific causes of death, those with SPD had an increased risk of mortality due to all CVDs, ischaemic heart disease, alcoholic liver disease and suicide, with the hazards ratios greatest for alcoholic liver disease and suicide mortality. However, no association was observed between SPD and mortality due to heart failure, cerebrovascular disease, all cancers, subtypes of cancers and diabetes mellitus. Given that sex-specific differences in life expectancy exist universally, we repeated the analyses for all-cause mortality stratifying by sex (see Supplementary Table 2). The risk of premature mortality was elevated for both males and females with SPD, albeit was slightly greater for males (HRs 1.68 vs 1.36). We did the same for age and smoking status and found similar patterns of elevated risk across all groups, except for those aged 46-65 years. Among those who did not meet physical activity recommendations, there was a statistically significant association between SPD and increased mortality risk. However, estimates did not greatly differ between those who did and did not meet recommendations (HR 1.30 vs 1.48, respectively). 
Table 2 Unadjusted HRs (and 95\% CIs) for all-cause and cause-specific mortality risk according to levels of psychological distress

\begin{tabular}{|c|c|c|c|c|c|}
\hline & \multicolumn{5}{|c|}{ Psychological distress levels, HRs (95\% CIs) } \\
\hline & Asymptomatic (0) & $\begin{array}{l}\text { Subclinical symptoms } \\
(1-5)\end{array}$ & Symptomatic (6-12) & $\begin{array}{l}\text { Highly symptomatic } \\
(13-24)\end{array}$ & $p$ trend \\
\hline Participants (N) & 97,381 & 69,745 & 22,776 & 5629 & \\
\hline \multicolumn{6}{|c|}{ All-cause mortality } \\
\hline Deaths $(N)$ & 3841 & 2470 & 1039 & 315 & \\
\hline Unadjusted & 1 & $0.86(0.81-0.91)$ & $1.18(1.09-1.27)$ & $1.58(1.37-1.83)$ & $<0.001$ \\
\hline \multicolumn{6}{|l|}{ All-CVD mortality } \\
\hline Deaths $(N)$ & 1151 & 753 & 316 & 101 & \\
\hline Unadjusted & 1 & $0.89(0.80-0.99)$ & $1.21(1.05-1.39)$ & $1.68(1.28-2.20)$ & 0.006 \\
\hline \multicolumn{6}{|c|}{ Ischaemic heart disease mortality } \\
\hline Deaths $(N)$ & 620 & 412 & 163 & 49 & \\
\hline Unadjusted & 1 & $0.90(0.79-1.03)$ & $1.14(0.93-1.40)$ & $1.58(1.09-2.30)$ & 0.145 \\
\hline \multicolumn{6}{|c|}{ Heart failure mortality } \\
\hline Deaths $(N)$ & 46 & 42 & 15 & 6 & \\
\hline Unadjusted & 1 & $1.32(0.81-2.13)$ & $1.61(0.85-3.03)$ & $2.69(1.12-6.48)$ & 0.002 \\
\hline \multicolumn{6}{|c|}{ Cerebrovascular disease mortality } \\
\hline Deaths $(N)$ & 188 & 133 & 48 & 13 & \\
\hline Unadjusted & 1 & $0.95(0.74-1.22)$ & $1.10(0.76-1.60)$ & $1.42(0.73-2.76)$ & 0.479 \\
\hline \multicolumn{6}{|c|}{ All-cancer related mortality } \\
\hline Deaths $(N)$ & 999 & 587 & 234 & 52 & \\
\hline Unadjusted & 1 & $0.79(0.70-0.90)$ & $0.94(0.80-1.11)$ & $0.93(0.67-1.28)$ & 0.047 \\
\hline \multicolumn{6}{|c|}{ Cancer of trachea, bronchus and lung mortality } \\
\hline Deaths $(N)$ & 342 & 182 & 86 & 23 & \\
\hline Unadjusted & 1 & $0.69(0.57-0.85)$ & $1.06(0.80-1.40)$ & $1.14(0.72-1.80)$ & 0.513 \\
\hline \multicolumn{6}{|c|}{ Cancer of colon, rectum and anus mortality } \\
\hline Deaths $(N)$ & 82 & 58 & 23 & 2 & \\
\hline Unadjusted & 1 & $0.81(0.56-1.16)$ & $1.08(0.65-1.79)$ & $0.58(0.14-2.41)$ & 0.556 \\
\hline \multicolumn{6}{|l|}{ Cancer of breast } \\
\hline Deaths $(N)$ & 55 & 31 & 14 & 7 & \\
\hline Unadjusted & 1 & $0.71(0.42-1.21)$ & $1.18(0.60-2.31)$ & $2.32(0.93-5.80)$ & 0.476 \\
\hline \multicolumn{6}{|l|}{ Cancer of prostate } \\
\hline Deaths $(N)$ & 39 & 12 & 7 & 1 & \\
\hline Unadjusted & 1 & $0.44(0.22-0.89)$ & $1.15(0.47-2.81)$ & $0.26(0.03-1.90)$ & 0.375 \\
\hline \multicolumn{6}{|c|}{ Diabetes mellitus mortality } \\
\hline Deaths $(N)$ & 38 & 26 & 8 & 5 & \\
\hline Unadjusted & 1 & $0.91(0.50-1.63)$ & $0.93(0.40-2.16)$ & $2.03(0.77-5.34)$ & 0.722 \\
\hline \multicolumn{6}{|c|}{ Alcoholic liver disease mortality } \\
\hline Deaths $(N)$ & 22 & 20 & 6 & 6 & \\
\hline Unadjusted & 1 & $1.13(0.54-2.37)$ & $0.78(0.30-2.05)$ & $4.63(1.65-12.98)$ & 0.185 \\
\hline \multicolumn{6}{|l|}{ Suicide mortality } \\
\hline Deaths $(N)$ & 58 & 43 & 18 & 11 & \\
\hline Unadjusted & 1 & $1.12(0.71-1.77)$ & $1.42(0.77-2.61)$ & $3.57(1.70-7.51)$ & 0.018 \\
\hline
\end{tabular}

CI, confidence intervals; HR, hazard ratio

\section{Discussion}

In this prospective cohort study of 195,531 US adults, PD symptoms of any severity were associated with increased risk of all-cause mortality over 5.9 years. This largely occurred in a dose-response manner and, when cause-specific mortality was examined, this was especially true for CVDs, ischaemic heart disease and heart failure. The hazards ratio for risk was highest for alcoholic liver disease and suicide-related mortality. However, only highly symptomatic 


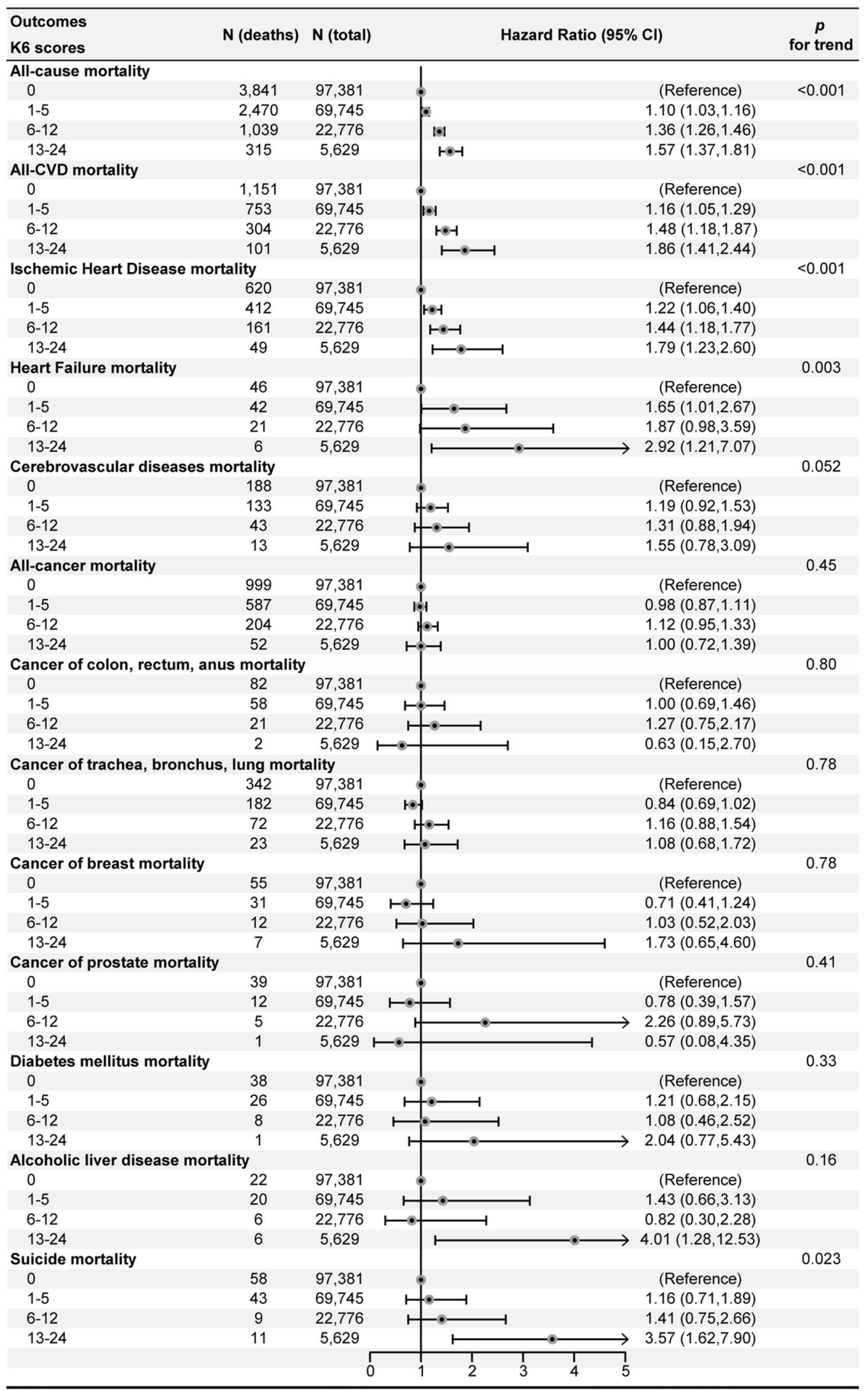

Fig. 1 Adjusted HRs (and 95\% CIs) for all-cause and cause-specific mortality risk according to levels of psychological distress 
Table 3 Adjusted HRs (and 95\% CIs) for all-cause and cause-specific mortality risk after excluding cases that occurred within the first 2 years of follow-up (sensitivity analysis)

Psychological distress levels, HRs (95\% CIs)
$\begin{aligned} & \text { Asymp- } \\ & \text { tomatic }\end{aligned}$

$p$ trend

tomatic

(0)

\begin{tabular}{|c|c|c|c|c|c|}
\hline \multicolumn{6}{|c|}{ All-cause mortality } \\
\hline Deaths $(N)$ & 3024 & 1931 & 788 & 239 & \\
\hline Adjusted HRs & 1 & $1.08(1.01-1.16)$ & $1.31(1.20-1.43)$ & $1.52(1.31-1.77)$ & $<0.001$ \\
\hline \multicolumn{6}{|c|}{ All-CVD mortality } \\
\hline Deaths $(N)$ & 888 & 575 & 239 & 82 & \\
\hline Adjusted HRs & 1 & $1.16(1.03-1.31)$ & $1.52(1.30-1.78)$ & $2.07(1.55-2.76)$ & $<0.001$ \\
\hline \multicolumn{6}{|c|}{ Ischaemic heart disease mortality } \\
\hline Deaths $(N)$ & 482 & 304 & 129 & 39 & \\
\hline Adjusted HRs & 1 & $1.14(0.98-1.34)$ & $1.50(1.20-1.88)$ & $1.98(1.30-3.02)$ & $<0.001$ \\
\hline \multicolumn{6}{|c|}{ Heart failure mortality } \\
\hline Deaths $(N)$ & 39 & 35 & 13 & 6 & \\
\hline Adjusted HRs & 1 & $1.76(1.05-2.96)$ & $2.23(1.13-4.41)$ & $3.93(1.65-9.37)$ & $<0.003$ \\
\hline \multicolumn{6}{|c|}{ Cerebrovascular disease mortality } \\
\hline Deaths $(N)$ & 153 & 104 & 35 & 10 & \\
\hline Adjusted HRs & 1 & $1.23(0.94-1.61)$ & $1.38(0.87-2.20)$ & $1.38(0.65-2.93)$ & 0.052 \\
\hline \multicolumn{6}{|c|}{ All-cancer related mortality } \\
\hline Deaths $(N)$ & 808 & 488 & 190 & 44 & \\
\hline Adjusted HRs & 1 & $1.02(0.89-1.17)$ & $1.13(0.94-1.35)$ & $1.03(0.72-1.47)$ & 0.317 \\
\hline \multicolumn{6}{|c|}{ Cancer of trachea, bronchus and lung mortality } \\
\hline Deaths $(N)$ & 277 & 152 & 70 & 17 & \\
\hline Adjusted HRs & 1 & $0.88(0.70-1.10)$ & $1.19(0.87-1.62)$ & $0.94(0.55-1.62)$ & 0.823 \\
\hline \multicolumn{6}{|c|}{ Cancer of colon, rectum and anus mortality } \\
\hline Deaths $(N)$ & 70 & 47 & 18 & 1 & \\
\hline Adjusted HRs & 1 & $0.98(0.65-1.47)$ & $1.10(0.60-2.04)$ & $0.42(0.06-3.15)$ & 0.761 \\
\hline \multicolumn{6}{|c|}{ Cancer of breast } \\
\hline Deaths $(N)$ & 44 & 25 & 12 & 7 & \\
\hline Adjusted HRs & 1 & $0.65(0.34-1.23)$ & $1.10(0.52-2.32)$ & $2.13(0.79-5.77)$ & 0.601 \\
\hline \multicolumn{6}{|c|}{ Cancer of prostate } \\
\hline Deaths $(N)$ & 34 & 9 & 4 & 1 & \\
\hline Adjusted HRs & 1 & $0.61(0.28-1.34)$ & $1.10(0.36-3.32)$ & $0.64(0.08-4.87)$ & 0.519 \\
\hline \multicolumn{6}{|c|}{ Diabetes mellitus mortality } \\
\hline Deaths $(N)$ & 30 & 21 & 6 & 5 & \\
\hline Adjusted HRs & 1 & $1.35(0.71-2.58)$ & $1.23(0.47-3.21)$ & $2.99(1.11-8.07)$ & 0.113 \\
\hline \multicolumn{6}{|c|}{ Alcoholic liver disease mortality } \\
\hline Deaths $(N)$ & 17 & 12 & 6 & 4 & \\
\hline Adjusted HRs & 1 & $0.76(0.34-1.73)$ & $0.89(0.31-2.59)$ & $2.63(0.63-10.99)$ & 0.629 \\
\hline \multicolumn{6}{|c|}{ Suicide mortality } \\
\hline Deaths $(N)$ & 41 & 28 & 9 & 4 & \\
\hline Adjusted HRs & 1 & $0.83(0.47-1.47)$ & $0.89(0.39-2.00)$ & $1.66(0.49-5.65)$ & 0.988 \\
\hline
\end{tabular}

Adjusted for age, sex, race, education, income, BMI, drinking alcohol, physical activity, smoking status, and survey year

CI, confidence intervals; HR, hazard ratio

levels of PD conferred a greater risk of mortality from these causes. In contrast, no association was observed between
PD and all cancers, subtypes of cancers, cerebrovascular diseases or diabetes mellitus in this cohort. 
To our knowledge, this study is the largest to date that considers the relative contributions of mortality due to allcauses, specific chronic disease subtypes and suicide across the spectrum of PD severity. Our findings are partially in keeping with other large prospective studies among those with PD. Cohort studies using data from the US NHIS $(N=330,367)[20]$ and the UK Biobank study $(N=308,721)$ [19] similarly observed positive dose-response associations between PD and all-cause and CVD-specific mortality. Similarly, a meta-analysis of 10 large prospective cohort studies $(N=68,222)$ from the Health Survey for England reported that PD was associated with an increased risk of mortality from all-causes, CVD, and external causes (e.g. injuries and poisonings) across all levels of distress. Our findings add to these studies by providing a more detailed analysis of specific-cause-related mortality among those with PD.

A key finding of this study was that we found no evidence of an association between PD and premature mortality due to all and specific types of cancers. This finding is in contrast to other large prospective studies that showed a positive association between PD symptoms and cancer mortality [20, 29]. This might relate to differences in measures of psychological distress used, length of follow-up or the overall proportion of cancer deaths within study populations. For example, the proportion of cancer deaths within our sample was lower than that of Russ et al. (1.0\% vs 3.7\% of deaths in the total sample, respectively). The number of deaths for certain cancers was also low in our sample, particularly among those with higher levels of distress. Therefore, we may be underpowered to detect an association if one existed.

We also found that those with subclinical PD symptoms were at increased risk of mortality due to CVD, but not suicide. The risk of mortality due to suicide was only evident among those with higher levels of distress, which is in line with previous studies [23, 34]. After removing cases that occurred within the initial 2 years of follow-up, PD symptoms, of any level, were not associated with suicide-specific mortality. However, this may be due to powering issues and a small number of cases, rather than a true absence of association. It is also possible that PD may have a more immediate impact on suicide risk when compared to chronic diseases, which typically worsen over time.

Our findings add to the existing evidence by quantifying the magnitude of associations between different thresholds of PD and cause-specific mortality with a focus on chronic disease versus suicide-related deaths. These data suggest that whilst CVD-related deaths are more frequent in those with PD compared to suicide, when considering all participants, the excess mortality risk owing to suicide is more pronounced in those with higher levels of $\mathrm{PD}$, than certain chronic diseases.

Our findings are biologically plausible and may aid our understanding of the pathways by which PD exerts an influence on disease-specific outcomes. Based on K10 validation studies against the Diagnostic and Statistical Manual of Mental Disorders and International Classification of Disease defined psychiatry disorders using the Composite International Diagnostic Interview, the correspondence between the K10 — and by extension-K6 scores and the probability of being identified as a current diagnostic case would suggest that PD in this study can be interpreted a more persistent distress [35]. Accumulating evidence indicates that persistent stress has a negative impact on cardiovascular, nervous and immune systems, and may increase susceptibility to numerous diseases [36]. PD can elicit changes to sympathetic and parasympathetic pathways [37], and dysregulation of the hypothalamic-pituitary-adrenal axis, which in turn may result in increased inflammation and cortisol release [38]. This may impact the cardiovascular system by precipitating myocardial infarction or dysrhythmia [37] or by accelerating the atherosclerotic process [37]. These changes are likely to occur over time with persistent levels of distress. Due to limitations in the data, we were unable to adjust for comorbid mental disorders, but it is plausible that at least some of the variation in the model is explained by mental health comorbidities or associated treatment regimens. For example, PD is highly comorbid with other psychiatric conditions including schizophrenia and bipolar disorder. Treatment regimens for these conditions (especially when treatment is refractory) have well-established cardiometabolic consequences or side effects [39]. These can be offset with lifestyle interventions at the time of initiation of therapy [40]. Among those not meeting physical activity recommendations, a significant association was observed between SPD and increased mortality risk. This association was not evident among those who met physical activity recommendations; however, the difference in estimates between the two groups was of a small magnitude. Nonetheless, even small effects may be of importance at the population level, given $100 \%$ population exposure.

Indeed, the association between PD and chronic diseaserelated mortality may be mediated by other behavioural and lifestyle factors. A study of adults from Scotland $(N=6576)$ previously found that the association between PD and CVD risk is largely explained by behavioral processes as increased smoking, physical inactivity and poor diet, which may be part of the coping response to PD and could potentiate disease pathogenesis [16]. However, these behaviors do not fully explain the poorer health outcomes among those with elevated PD with drivers of these disparities including healthcare utilization, social barriers and the healthcare system. For instance, those with mental disorders often have suboptimal access to services and experience isolation and socioeconomic disadvantage, which may lead to poorer health outcomes [41]. In addition, gaps in current services may mean that physical conditions go undiagnosed 
and untreated in those with mental disorders. For example, those with mental disorders are less likely to be offered standard physical health checks and routine screening for chronic conditions [41, 42], as mental health presentations often overshadow physical health needs [43]. Diagnostic and treatment overshadowing, whereby treatment is targeted towards managing mental health symptoms rather than on comorbid physical health conditions [44], can result in worse physical health outcomes for those with mental disorders, which may ultimately lead to premature mortality [44].

Strengths of this study include the use of a large, population-based sample that was linked to a centralized database of all US deaths which provided data for cause-specific mortality. We also investigated different levels of PD, which enabled us to examine the dose-response relationship, in addition to the binary classification of distress. This is in line with recommendations to test more than one threshold for classifying cases [45]. Our study adjusted for numerous sociodemographic and lifestyle factors that are known to be associated with mortality. For robustness, we further stratified analyses to address residual confounding by age, sex, smoking status and physical activity. It should be noted, however, that large-scale, population-based studies may be subject to residual confounding as a result of unmeasured or poorly measured confounders. If pre-existing diseases explained the observed increases in mortality rates, it could be expected that a larger number of deaths would occur within the initial follow-up period. However, to minimize the risk of reverse causation, we excluded deaths that occurred within the first 2 years of follow-up and observed similar findings to that of the main study findings for chronic diseases, which suggests that other mechanisms are likely to be driving these associations.

Our findings should be interpreted in light of the study limitations. First, the K6 scale used to assess psychological symptoms was designed as a screening tool rather than a diagnostic device and is reliant on self-report data. Using this tool, we were unable to distinguish between PD and the presence of major depression. Therefore, caution should be used when drawing comparisons with studies that have assessed depression symptoms with validated clinical assessments. Second, PD symptoms are non-specific and may overlap with other mental disorders. As such, the presence of other psychiatric conditions that are reflected in distress may have influenced our risk estimates. Third, whilst our finding of increased risk of suicide mortality may be a true phenomenon, there is a possibility that this is a spurious finding based on a reasonably small number of suicide cases, which may have inflated HRs. Moreover, we may have been underpowered to detect differences in mortality owing to suicide across all four levels of PD. This may also be true for mortality due to other cause-specific outcomes (e.g. cerebrovascular disease and diabetes) whereby too few deaths may have occurred within higher levels of distress to detect a significant association. Therefore, future large-scale studies are required to corroborate these findings.

The prevention and treatment of mental disorders is an important public health priority, especially in the current COVID-19 setting. Suicide prevention is currently at the forefront of health promotion strategies for PD, particularly among those with higher symptoms of distress [46]. However, the increased risk of mortality attributable to chronic diseases in this study suggests that chronic disease prevention strategies also warrant attention. Our findings suggest that interventions aimed at improving physical health outcomes should be targeted towards those at lower levels of distress than currently recommended for suicide prevention (i.e. mild and moderate distress symptoms) [46]. Identifying people who are more vulnerable to the health consequences of PD through systematic screening (e.g. using the K6 scale) may improve the recognition of early PD and provide opportunities for early intervention [47]. In doing so, this may not only reduce the risk of clinically diagnosed disorders (e.g. major depression) [48] but also the risk of premature mortality. Future research should focus on the improvement of screening and development of interventions (e.g. diet, smoking and physical activity interventions) [2] aimed at mutually improving mental and physical health outcomes in those with PD. In turn, this may reduce the risk of resulting premature mortality.

\section{Conclusions}

In summary, our findings suggest that PD symptoms, of any level, were associated with an increased risk of allcause mortality. In particular, we observed a dose-response association between PD and mortality due to all CVDs and specific disease subtypes. Notably, those with low levels of distress were at an increased risk of mortality due to certain chronic diseases, but not suicide. These findings highlight that physical health disparities contribute to premature mortality and emphasize the need for further research into lifestyle interventions targeted towards improving physical health outcomes among those with PD.

Supplementary Information The online version contains supplementary material available at https://doi.org/10.1007/s00127-021-02116-7.

Acknowledgements This research received no specific grant from any funding agency, commercial or not-for-profit sectors. MH is supported by an Australian Rotary Health PhD Scholarship. TR has been supported by the Fernwood Foundation. AR has a position as a university lecturer at University of Eastern Finland and as a postdoctoral researcher at Kuopio University Hospital, Finland. AO is supported by a Future Leader Fellowship (\#101160) from the Heart Foundation Australia and Wilson Foundation. 
Author contributions All authors contributed to the study conception and design. Material preparation, data collection and analysis were performed by WH, BL, and JN. The first draft of the manuscript was written by $\mathrm{MH}$ and all authors commented on previous versions of the manuscript. All authors read and approved the final manuscript.

Funding This research received no specific grant from any funding agency in the public, commercial, or not-for-profit sectors.

Availability of data and materials The data analysed during the current study are available from the corresponding author.

\section{Code availability Not applicable.}

\section{Declarations}

Conflict of interest TR has received grants, fellowships and research support from University of the Sunshine Coast, Australian Postgraduate Awards, Fernwood Foundation and Be Fit Food. TR received consultancy, honoraria and travel funds from Oxford University Press, the University of Melbourne, the University of Sydney, Bond University, University of Southern Queensland, Dietitians Association of Australia, Nutrition Society of Australia, The Royal Australian and New Zealand College of Psychiatrists, Academy of Nutrition and Dietetics, Black Dog Institute, Australian Rotary Health, Australian Disease Management Association, Department of Health and Human Services, Primary Health Networks, Barwon Health, West Gippsland Healthcare Group, Central West Gippsland Primary Care Partnership, Parkdale College, City of Greater Geelong and Global Age. FNJ has received: (1) competitive Grant/Research support from the Brain and Behaviour Research Institute, the National Health and Medical Research Council (NHMRC), Australian Rotary Health, the Geelong Medical Research Foundation, the Ian Potter Foundation, The University of Melbourne; (2) industry support for research from Meat and Livestock Australia, Woolworths Limited, the A2 Milk Company, Be Fit Foods; (3) philanthropic support from the Fernwood Foundation, Wilson Foundation, the JTM Foundation, the Serp Hills Foundation, the Roberts Family Foundation, the Waterloo Foundation; and (4) travel support and speakers honoraria from Sanofi-Synthelabo, Janssen Cilag, Servier, Pfizer, Network Nutrition, Angelini Farmaceutica, Eli Lilly and Metagenics. FNJ has written two books for commercial publication. AO has received research funding from National Health \& Medical Research Council, Australian Research Council, University of Melbourne, Deakin University, Sanofi, Meat and Livestock Australia and Woolworths Limited and Honoraria from Novartis. For the remaining authors, no conflicts of interest were declared.

\section{References}

1. Whiteford HA, Ferrari AJ, Degenhardt L, Feigin V, Vos T (2015) The global burden of mental, neurological and substance use disorders: an analysis from the Global Burden of Disease Study 2010. PLoS One 10(2):e0116820. https://doi.org/10.1371/journ al.pone. 0116820

2. Firth J, Siddiqi N, Koyanagi A, Siskind D, Rosenbaum S, Galletly C, Allan S, Caneo C, Carney R, Carvalho AF, Chatterton ML, Correll CU, Curtis J, Gaughran F, Heald A, Hoare E, Jackson SE, Kisely S, Lovell K, Maj M, McGorry PD, Mihalopoulos C, Myles H, O'Donoghue B, Pillinger T, Sarris J, Schuch FB, Shiers D, Smith L, Solmi M, Suetani S, Taylor J, Teasdale SB, Thornicroft G, Torous J, Usherwood T, Vancampfort D, Veronese N, Ward PB, Yung AR, Killackey E, Stubbs B (2019) The Lancet
Psychiatry Commission: a blueprint for protecting physical health in people with mental illness. Lancet Psychiatry 6(8):675-712. https://doi.org/10.1016/s2215-0366(19)30132-4

3. O'Neil A, Stevenson C, Williams E, Mortimer D, Oldenburg B, Sanderson K (2013) The Health Related Quality of Life burden of co-morbid Cardiovascular Disease and major depressive disorder in Australia. Findings from a population-based, cross sectional study. Qual Life Res 22(1):37-44

4. O'Neil A, Williams E, Stevenson C, Oldenburg B, Sanderson K (2012) Co-morbid depression is associated with poor work outcomes in persons with cardiovascular disease (CVD): a large, nationally representative survey in the Australian population. BMC Public Health 12:47

5. Australian Bureau of Statistics (2016) National health survey: mental health and coexisting physical health conditions, 2014-15. Australian Bureau of Statistics, Australia. https://www.abs.gov. au/ausstats/abs@.nsf/0/C0A4290EF1E7E7FDCA257F1E001C0B 84? Opendocument. Accessed 24 July 2020

6. World Health Organization (2015) Meeting report on excess mortality in persons with severe mental disorders. World Health Organisation. http://www.who.int/mental_health/evidence/ excess_mortality_meeting_report.pdf. Accessed 24 July 2020

7. Chesney EG, Goodwin M, Fazel S (2014) Risks of all-cause and suicide mortality in mentaldisorders: a meta-review. World Psychiatry $13: 153-160$

8. Naghavi M, Global Burden of Disease Self-Harm C (2019) Global, regional, and national burden of suicide mortality 1990 to 2016: systematic analysis for the Global Burden of Disease Study 2016. BMJ 364:194. https://doi.org/10.1136/bmj.194

9. Lawrence D, Hancock K, Kisely S (2013) The gap in life expectancy from preventable physical illness in psychiatric patients in Western Australia: retrospective analysis of population based registers. Br Med J 346:f2539

10. Harris B, Duggan M, Batterham P, Bartlem K, Clinton-McHarg T, Dunbar J, Fehily C, Lawrence D, Morgan M, Rosenbaum S (2018) Australia's Mental and Physical Health Tracker: background paper. Australian Health Policy Collaboration issues paper no. 2018-02, Melbourne, AHPC. p 53

11. Mykletun A, Bjerkeset O, Dewey M, Prince M, Overland S, Stewart R (2007) Anxiety, depression, and cause-specific mortality: the HUNT study. Psychosom Med 69(4):323-331. https://doi.org/10. 1097/PSY.0b013e31803cb862

12. Colman I, Kingsbury M, Sucha E, Horton NJ, Murphy JM, Gilman SE (2018) Depressive and anxious symptoms and 20-year mortality: evidence from the Stirling County study. Depress Anxiety 35(7):638-647. https://doi.org/10.1002/da.22750

13. Mykletun A, Bjerkeset O, Overland S, Prince M, Dewey M, Stewart R (2009) Levels of anxiety and depression as predictors of mortality: the HUNT study. Br J Psychiatry 195(2):118-125. https://doi.org/10.1192/bjp.bp.108.054866

14. Arvidsdotter T, Marklund B, Kylen S, Taft C, Ekman I (2016) Understanding persons with psychological distress in primary health care. Scand J Caring Sci 30(4):687-694. https://doi.org/ 10.1111/scs. 12289

15. Cuijpers P, Smit F (2002) Excess mortality in depression: a metaanalysis of community studies. J Affect Disord 72:227-236

16. Hamer M, Molloy GJ, Stamatakis E (2008) Psychological distress as a risk factor for cardiovascular events: pathophysiological and behavioral mechanisms. J Am Coll Cardiol 52(25):2156-2162. https://doi.org/10.1016/j.jacc.2008.08.057

17. Stansfeld S, Fuhrer R, Shipley M, Marmot M (2002) Psychological distress as a risk factor for coronary heart disease in the Whitehall II Study. Int J Epidemiol 31:248-255

18. Eriksson AK, Ekbom A, Granath F, Hilding A, Efendic S, Ostenson CG (2008) Psychological distress and risk of pre-diabetes and Type 2 diabetes in a prospective study of Swedish middle-aged 
men and women. Diabet Med 25(7):834-842. https://doi.org/10. 1111/j.1464-5491.2008.02463.x

19. Batty GD, McIntosh AM, Russ TC, Deary IJ, Gale CR (2016) Psychological distress, neuroticism, and cause-specific mortality: early prospective evidence from UK Biobank. J Epidemiol Community Health 70(11):1136-1139. https://doi.org/10.1136/ jech-2016-207267

20. Yang L, Zhao M, Magnussen CG, Veeranki SP, Xi B (2020) Psychological distress and mortality among US adults: prospective cohort study of 330367 individuals. J Epidemiol Community Health 74(4):384-390. https://doi.org/10.1136/jech-2019-213144

21. Hamer M, Chida Y, Molloy GJ (2009) Psychological distress and cancer mortality. J Psychosom Res 66(3):255-258. https://doi.org/ 10.1016/j.jpsychores.2008.11.002

22. Russ TC, Kivimaki M, Morling JR, Starr JM, Stamatakis E, Batty GD (2015) Association between psychological distress and liver disease mortality: a meta-analysis of individual study participants. Gastroenterology 148(5):958-966. https://doi.org/10.1053/j.gastro.2015.02.004 ((e954))

23. Walker E, McGee R, Druss B (2015) Mortality in mental disorders and global disease burden implications: a systematic review and meta-analysis. JAMA Psychiat 72(4):334-341. https://doi.org/10. 1001/jamapsychiatry.2014.2502

24. Centers for Disease Control and Prevention (2020) National Health Interview Survey. CDC. https://www.cdc.gov/nchs/nhis/ index.htm. Accessed Sept 2020

25. Blewett L, Rivera Drew J, King M, Williams K (2019) IPUMS Health Surveys: National Health Interview Survey 6.4 edn. Minneapolis, MN. https://doi.org/10.18128/D070.V6.4

26. National Center for Health Statistics Office of Analysis and Epidemiology (2019) The linkage of National Center for Health Statistics survey data to the National Death Index-2015 linked mortality file (LMF): methodology overview and analytic considerations. National Center for Health Statistics. https://www.cdc.gov/nchs/ data-linkage/mortality-methods.htm. Accessed 1 June 2019

27. Kessler RCBP, Colpe LJ, Epstein JF, Gfroerer JC, Hiripi E et al (2003) Screening for serious mental illness in the general population. Arch Gen Psychiatry 60:184-189

28. Prochaska JJ, Sung HY, Max W, Shi Y, Ong M (2012) Validity study of the K6 scale as a measure of moderate mental distress based on mental health treatment need and utilization. Int J Methods Psychiatr Res 21(2):88-97. https://doi.org/10.1002/mpr.1349

29. Russ TC, Stamatakis E, Hamer M, Starr JM, Kivimaki M, Batty GD (2012) Association between psychological distress and mortality: individual participant pooled analysis of 10 prospective cohort studies. BMJ 345:e4933. https://doi.org/10.1136/bmj. e4933

30. Batty GD, Russ TC, Stamatakis E, Kivimaki M (2017) Psychological distress in relation to site specific cancer mortality: pooling of unpublished data from 16 prospective cohort studies. BMJ 356:j108. https://doi.org/10.1136/bmj.j108

31. Pratt LA (2009) Serious psychological distress, as measured by the K6, and mortality. Ann Epidemiol 19(3):202-209. https://doi. org/10.1016/j.annepidem.2008.12.005

32. Streiner D, Cairney J (2007) What's under the ROC? An introduction to receiver operating characteristics curves. Can J Psychiatry 52(2):121-128

33. Lochner K, Hummer RA, Bartee S, Wheatcroft G, Cox C (2008) The public-use National Health Interview Survey linked mortality files: methods of reidentification risk avoidance and comparative analysis. Am J Epidemiol 168(3):336-344. https://doi.org/10. 1093/aje/kwn123
34. Bell S, Russ TC, Kivimaki M, Stamatakis E, Batty GD (2015) Dose-response association between psychological distress and risk of completed suicide in the general population. JAMA Psychiat 72(12):1254-1256

35. Andrews GST (2001) Interpreting scores on the Kessler Psychological Distress Scale (K10). Aust N Z J Public Health 25:494-497

36. Juster R-P, McEwen BS, Lupien SJ (2010) Allostatic load biomarkers of chronic stress and impact on health and cognition. Neurosci Biobehav Rev 35(1):2-16

37. Brotman DJ, Golden SH, Wittstein IS (2007) The cardiovascular toll of stress. Lancet 370(9592):1089-1100. https://doi.org/10. 1016/s0140-6736(07)61305-1

38. Dinan T (2009) Inflammatory markers in depression. Curr Opin Psychiatry 22(1):32-36

39. Hyde N, Dodd S, Venugopal K, Purdie C, Berk M, O'Neil A (2015) Prevalence of cardiovascular and metabolic events in patients prescribed clozapine: a retrospective observational, clinical cohort study. Curr Drug Saf 10(2):125-131

40. Curtis J, Watkins A, Rosenbaum S, Teasdale S, Kalucy M, Samaras K, Ward PB (2016) Evaluating an individualized lifestyle and life skills intervention to prevent antipsychotic-induced weight gain in first-episode psychosis. Early Interv Psychiatry 10(3):267-276. https://doi.org/10.1111/eip.12230

41. Lawrence D, Kisely S (2010) Inequalities in healthcare provision for people with severe mental illness. J Psychopharmacol 24(4 Suppl):61-68. https://doi.org/10.1177/1359786810382058

42. de Hert M, Cohen D, Bobes J, Cetokovich-Bakmas M, Leucht S, Ndetei DM, Newcomer JW, Uwakwe R, Asai I, Moller H-J (2011) Physical illness in patients with severe mental disorders. II. Barriers to care, monitoring and treatment guidelines, plus recommendations at the system and individual level. World Psychiatry 10(2):138-151

43. World Health Organisation (2018) Management of physical health conditions in adults with severe mental disorders: WHO guidelines. World Health Organisation, Geneva

44. Jones S, Howard L, Thornicroft G (2008) "Diagnostic overshadowing": worse physical health care for people with mental illness. Acta Psychiatr Scand 118(3):169-171. https://doi.org/10.1111/j. 1600-0447.2008.01211.x

45. Ferraro K, Nuriddin T (2006) Psychological distress and mortality: are women more vulnerable? J Health Soc Behav 47(3):227-241

46. Zalsman G, Hawton K, Wasserman D, van Heeringen K, Arensman E, Sarchiapone M, Carli V, Höschl C, Barzilay R, Balazs J, Purebl G, Kahn JP, Sáiz PA, Lipsicas CB, Bobes J, Cozman D, Hegerl U, Zohar J (2016) Suicide prevention strategies revisited: 10-year systematic review. Lancet Psychiatry 3(7):646-659. https://doi.org/10.1016/s2215-0366(16)30030-x

47. Lazzarino AI, Hamer M, Stamatakis E, Steptoe A (2013) The combined association of psychological distress and socioeconomic status with all-cause mortality: a national cohort study. JAMA Intern Med 173(1):22-27. https://doi.org/10.1001/2013. jamainternmed.951

48. Karsten J, Hartman CA, Smit JH, Zitman FG, Beekman AT, Cuijpers P, van der Does AJ, Ormel J, Nolen WA, Penninx BW (2011) Psychiatric history and subthreshold symptoms as predictors of the occurrence of depressive or anxiety disorder within 2 years. Br J Psychiatry 198(3):206-212. https://doi.org/10.1192/bjp.bp. 110.080572 